PROCEEDINGS OF THE

AMERICAN MATHEMATICAL SOCIETY

Volume 128, Number 7, Pages 2105-2109

S 0002-9939(00)05246-1

Article electronically published on February 25, 2000

\title{
A NEW CHARACTERIZATION OF MÖBIUS TRANSFORMATIONS BY USE OF APOLLONIUS HEXAGONS
}

\author{
HIROSHI HARUKI AND THEMISTOCLES M. RASSIAS
}

(Communicated by David R. Larson)

\begin{abstract}
The purpose of this paper is to give a new characterization of Möbius transformations from the standpoint of conformal mappings. To this end a new concept of Apollonius hexagons on the complex plane is used.
\end{abstract}

\section{INTRODUCTION}

In 3. a new characterization of Möbius (that is, linear rational) transformations among conformal mappings was given. We considered Apollonius quadrilaterals, that is, not necessarily simple quadrilaterals for which $\overline{A B} \cdot \overline{C D}=\overline{B C} \cdot \overline{D A}$ (the bar indicates the length of the segment) and showed that Möbius transformations are the only conformal mappings which conserve this equation.

In the present paper we consider Apollonius hexagons.

Definition 1. A hexagon $A B C D E F$ (not necessarily simple) on the complex plane for which $\overline{A B} \cdot \overline{C D} \cdot \overline{E F}=\overline{B C} \cdot \overline{D E} \cdot \overline{F A}$ holds (where the bar denotes the length of the segment) is an Apollonius hexagon.

Example 1. If one of the following conditions holds, then $A B C D E F$ is trivially an Apollonius hexagon.

(i) $\overline{A B}=\overline{A F}, \overline{C B}=\overline{C D}$ and $\overline{E D}=\overline{E F}$,

(ii) $\overline{A B}=\overline{D E}, \overline{B C}=\overline{E F}$ and $\overline{C D}=\overline{F A}$,

(iii) $\overline{A B}=\overline{B C}=\overline{C D}=\overline{D E}=\overline{E F}=\overline{F A}$,

(iv) $A B C D E F$ is a regular hexagon.

Example 2. Let $A B C D E F$ be a hexagon inscribed in a circle of the complex plane. Then $A B C D E F$ is an Apollonius hexagon iff the three diagonals $A D, B E$, and $C F$ are concurrent (cf. [5, p. 137]).

Example 3. Let $\triangle A B C$ be a triangle in the complex plane with angles $3 \alpha, 3 \beta, 3 \gamma$ at $A, B, C$, respectively. Draw lines from $A$ at the angle $\alpha$ and from $B$ at the angle $\beta$ outside the triangle, away from the side $A B$. Denote their point of intersection by $P$. The points $Q$ (from $B C$ ) and $R$ (from $C A$ ) are constructed similarly; so we get the Morley triangle $\triangle P Q R$ (cf. [1, pp. 47-49]). The hexagon $A R B P C Q$ is an Apollonius hexagon.

Received by the editors February 4, 1998 and, in revised form, June 29, 1998 and September 3, 1998.

1991 Mathematics Subject Classification. Primary 39B40; Secondary 33A70.

Key words and phrases. Möbius transformations, conformal mapping, Apollonius hexagons.

(C)2000 American Mathematical Society 
Proof. Applying the Sine Law of trigonometry in $\triangle P B C, \triangle Q C A$ and $\triangle R A B$ yields

$$
\frac{\overline{B P}}{\overline{P C}}=\frac{\sin \gamma}{\sin \beta}, \quad \frac{\overline{C Q}}{\overline{Q A}}=\frac{\sin \alpha}{\sin \gamma}, \quad \frac{\overline{A R}}{\overline{R B}}=\frac{\sin \beta}{\sin \alpha} .
$$

Multiplying the above three equalities gives

and therefore

$$
\frac{\overline{B P}}{\overline{P C}} \cdot \frac{\overline{C Q}}{\overline{Q A}} \cdot \frac{\overline{A R}}{\overline{R B}}=1
$$

$$
\overline{A R} \cdot \overline{B P} \cdot \overline{C Q}=\overline{R B} \cdot \overline{P C} \cdot \overline{Q A} .
$$

Hence, $A R B P C Q$ is an Apollonius hexagon.

Property A. Suppose that $f$ is analytic and univalent on a nonempty open region $\triangle$ on the complex plane. Let $A B C D E F$ be an Apollonius hexagon in $\Delta$. If we set $Z^{\prime}=f(Z)(Z=A, B, C, D, E, F)$, then $A^{\prime} B^{\prime} C^{\prime} D^{\prime} E^{\prime} F^{\prime}$ is also an Apollonius hexagon. We want to find all functions which have Property $\mathrm{A}$

The purpose of this paper is to prove the following theorem:

Theorem. $w=f(z)$ satisfies Property $\triangle$ iff $w=f(z)$ is a Möbius transformation of the variable $z$.

\section{Proof of the Theorem}

If. Substitution shows that all Möbius transformations, given by

$$
f(z)=\frac{a z+b}{c z+d}
$$

where $a, b, c, d$ are complex constants satisfying $a d \neq b c$ have the Property $\mathrm{A}$

Only if. By hypothesis, $f$ is analytic and univalent on the open region $\Delta$. Hence, by a well-known theorem (cf. [2, p. 56]) we obtain

$$
f^{\prime}(z) \neq 0
$$

on $\Delta$. Since $\Delta$ is an open region, for any of its points $x$ there exists a closed circular neighborhood $U$ (of radius $r$, say) such that (2) holds on $U$.

We consider an arbitrary regular hexagon $A B C D E F$ contained in $U$ where the sense of $A, B, C, D, E, F$ is counterclockwise and whose centre is at $x$. Therefore, $A, B, C, D, E, F$ can be represented by the complex numbers

$$
x+y, \quad x-\omega^{2} y, \quad x+\omega y, \quad x-y, \quad x+\omega^{2} y, \quad x-\omega y,
$$

respectively, where $0<|y| \leq r$ and $\omega=\frac{-1+i \sqrt{3}}{2}$. Let the circumscribed circle of $A B C D E F$ be $K$. We shall prove that if we set $A^{\prime}=f(A), B^{\prime}=f(B), C^{\prime}=$ $f(C), D^{\prime}=f(D), E^{\prime}=f(E)$ and $F^{\prime}=f(F)$, then $A^{\prime} C^{\prime} D^{\prime} E^{\prime}$ is an Apollonius quadrilateral, i.e.,

$$
\begin{aligned}
& |f(x+y)-f(x+\omega y)| \cdot\left|f(x-y)-f\left(x+\omega^{2} y\right)\right| \\
& \quad=|f(x+\omega y)-f(x-y)| \cdot\left|f\left(x+\omega^{2} y\right)-f(x+y)\right|
\end{aligned}
$$

holds on the $w$-plane.

To this end we take two variable points $B_{1}$ and $F_{1}$ on the open arc $A B$ (excluding $A, B)$ of $K$ and the open arc $A F$ (excluding $A, F)$ of $K$, respectively, such that

$$
\overline{A B}_{1}=\overline{A F}_{1} \text {. }
$$


By (4) and by $\overline{A B}=\overline{A F}$ we have

$$
\overline{B_{1} C}=\overline{F_{1} E}
$$

Since $A B C D E F$ is a regular hexagon, we obtain

$$
\overline{C D}=\overline{D E} \text {. }
$$

By (41), (5) and (6) we get

$$
\overline{A B_{1}} \cdot \overline{C D} \cdot \overline{E F_{1}}=\overline{B_{1} C} \cdot \overline{D E} \cdot \overline{F_{1} A} .
$$

Hence by definition the hexagon $A B_{1} C D E F_{1}$ is an Apollonius hexagon.

We set $B_{1}^{\prime}=f\left(B_{1}\right)$ and $F_{1}^{\prime}=f\left(F_{1}\right)$. Since, by hypothesis, $w=f(z)$ satisfies Property $\mathbb{A}$, we obtain

$$
\overline{A^{\prime} B_{1}^{\prime}} \cdot \overline{C^{\prime} D^{\prime}} \cdot \overline{E^{\prime} F_{1}^{\prime}}=\overline{B_{1}^{\prime} C^{\prime}} \cdot \overline{D^{\prime} E^{\prime}} \cdot \overline{F_{1}^{\prime} A^{\prime}}
$$

on the $w$-plane.

By (4), $B_{1}$ and $F_{1}$ can be represented by complex numbers

$$
x+e^{i \theta} y \text { and } x+e^{-i \theta} y,
$$

respectively, where $\theta$ is a real number satisfying $0<\theta<\frac{\pi}{3}$.

Since

$$
\begin{aligned}
& \overline{A^{\prime} B_{1}^{\prime}}=\left|f(x+y)-f\left(x+e^{i \theta} y\right)\right|, \quad \overline{C^{\prime} D^{\prime}}=|f(x+\omega y)-f(x-y)|, \\
& \overline{E^{\prime} F_{1}^{\prime}}=\left|f\left(x+\omega^{2} y\right)-f\left(x+e^{-i \theta} y\right)\right|, \quad \overline{B_{1}^{\prime} C}=\left|f\left(x+e^{i \theta} y\right)-f(x+\omega y)\right|, \\
& \overline{D^{\prime} E^{\prime}}=\left|f(x-y)-f\left(x+\omega^{2} y\right)\right|, \quad \overline{F_{1}^{\prime} A^{\prime}}=\left|f\left(x+e^{-i \theta} y\right)-f(x+y)\right|
\end{aligned}
$$

hold on the $w$-plane, by (7) we obtain

(8)

$$
\begin{aligned}
& \left|f(x+y)-f\left(x+e^{i \theta} y\right)\right||f(x+\omega y)-f(x-y)|\left|f\left(x+\omega^{2} y\right)-f\left(x+e^{-i \theta} y\right)\right| \\
& \quad=\left|f\left(x+e^{i \theta} y\right)-f(x+\omega y)\right|\left|f(x-y)-f\left(x+\omega^{2} y\right)\right|\left|f\left(x+e^{-i \theta} y\right)-f(x+y)\right| .
\end{aligned}
$$

Since the two points $x+e^{-i \theta} y, x+y$ are different points belonging to $U$ and $U$ is a subset of $\Delta, x+e^{-i \theta} y$ and $x+y$ are different points of $\Delta$. By hypothesis $w=f(z)$ is univalent in $\Delta$. So we obtain

$$
f\left(x+e^{-i \theta} y\right)-f(x+y) \neq 0 .
$$

By (8), (9) we get

$$
\begin{aligned}
& \left|\frac{f(x+y)-f\left(x+e^{i \theta} y\right)}{f\left(x+e^{-i \theta} y\right)-f(x+y)}(f(x+\omega y)-f(x-y))\left(f\left(x+\omega^{2} y\right)-f\left(x+e^{-i \theta} y\right)\right)\right| \\
& \quad=\left|\left(f\left(x+e^{i \theta} y\right)-f(x+\omega y)\right)\left(f(x-y)-f\left(x+\omega^{2} y\right)\right)\right| .
\end{aligned}
$$

If we let $\theta \rightarrow+0$, then

$$
\frac{f(x+y)-f\left(x+e^{i \theta} y\right)}{f\left(x+e^{-i \theta} y\right)-f(x+y)}
$$

is an indeterminate form.

Furthermore, since $x+y \in U$, we obtain

$$
f^{\prime}(x+y) \neq 0 .
$$


By (11) we obtain

$$
\lim _{\theta \rightarrow+0} \frac{f(x+y)-f\left(x+e^{-i \theta} y\right)}{f\left(x+e^{-i \theta} y\right)-f(x+y)}=\lim _{\theta \rightarrow+0} \frac{-i e^{i \theta} y f^{\prime}\left(x+e^{i \theta} y\right)}{-i e^{-i \theta} y f^{\prime}\left(x+e^{-i \theta} y\right)}=\frac{f^{\prime}(x+y)}{f^{\prime}(x+y)}=1 .
$$

Letting $\theta \rightarrow+0$ in (10) and using (12) yields

$$
\begin{aligned}
& \left|(f(x+\omega y)-f(x-y))\left(f\left(x+\omega^{2} y\right)-f(x+y)\right)\right| \\
& \quad=\left|(f(x+y)-f(x+\omega y))\left(f(x-y)-f\left(x+\omega^{2} y\right)\right)\right| .
\end{aligned}
$$

Hence $A^{\prime} C^{\prime} D^{\prime} E^{\prime}$ is an Apollonius quadrilateral.

Now we are ready to show that $f$ is a Möbius transformation. By (13) we have

$$
\left|\frac{(f(x+\omega y)-f(x-y))\left(f\left(x+\omega^{2} y\right)-f(x+y)\right)}{(f(x+y)-f(x+\omega y))\left(f(x-y)-f\left(x+\omega^{2} y\right)\right)}\right|=1 .
$$

If we set

$$
g(y)=\frac{(f(x+\omega y)-f(x-y))\left(f\left(x+\omega^{2} y\right)-f(x+y)\right)}{(f(x+y)-f(x+\omega y))\left(f(x-y)-f\left(x+\omega^{2} y\right)\right)}
$$

then, by (14), we have

$$
|g(y)|=1 \text {. }
$$

Since the numerator and the denominator of $g(y)$ in (15) are analytic for all $y$ satisfying $0<|y| \leq r$ and since, by the fact that $w=f(z)$ is univalent in $\Delta$, the denominator of $g(y)$ in (15) never vanishes in $0<|y| \leq r$.

Next, we shall prove that $g(y)$ is also analytic at $y=0$. As $y \rightarrow 0$, we have

$$
\frac{f(x+\omega y)-f(x-y)}{f(x+y)-f(x+\omega y)} \rightarrow \frac{\omega f^{\prime}(x)+f^{\prime}(x)}{f^{\prime}(x)-\omega f^{\prime}(x)}=\frac{1+\omega}{1-\omega}
$$

and

$$
\frac{f\left(x+\omega^{2} y\right)-f(x+y)}{f(x-y)-f\left(x+\omega^{2} y\right)} \rightarrow \frac{\omega^{2} f^{\prime}(x)-f^{\prime}(x)}{-f^{\prime}(x)-\omega^{2} f^{\prime}(x)}=\frac{1-\omega^{2}}{1+\omega^{2}} .
$$

Hence, by (15), (17) and (18), as $y \rightarrow 0$, one has

$$
g(y) \rightarrow\left(\frac{1+\omega}{1-\omega}\right)\left(\frac{1-\omega^{2}}{1+\omega^{2}}\right)=-1
$$

If we define

$$
g(0)=-1
$$

by (19), then, by Riemann's Theorem on removable singularities (cf. [2, p. 260]), the function $g(y)$ is analytic at $y=0$. Furthermore, by (20), the equality (16) still holds at $y=0$.

Summarizing. $g$ is analytic and its absolute value is 1 on a closed neighborhood (of radius $r$ ) of 0 . Therefore, by the maximum modulus theorem for analytic functions (cf. [2, p. 201]), we have on this neighborhood,

$$
g(y)=L
$$

where $L$ is a complex constant.

By (20) we get

$$
L=-1 \text {. }
$$


By (15), (21) and (22) we have

$$
\begin{aligned}
& (f(x+\omega y)-f(x-y))\left(f\left(x+\omega^{2} y\right)-f(x+y)\right) \\
& \quad+(f(x+y)-f(x+\omega y))\left(f(x-y)-f\left(x+\omega^{2} y\right)\right)=0
\end{aligned}
$$

for all $y$ satisfying $|y| \leq r$. Differentiating both sides of (23) four times with respect to $y$, setting $y=0$ and simplifying the resulting equality yields

$$
f^{\prime \prime \prime}(x) f^{\prime}(x)-\frac{3}{2} f^{\prime \prime}(x)^{2}=0
$$

Since $x \in \Delta$ was arbitrarily fixed, we can replace $x$ by a variable $z$ and thus, by (24), we have

$$
f^{\prime \prime \prime}(z) f^{\prime}(z)-\frac{3}{2} f^{\prime \prime}(z)^{2}=0
$$

in $\Delta$. By the Uniqueness Theorem (cf. [2] p. 242]) the above equality holds in $|z|<+\infty$.

Hence we obtain

$$
\frac{f^{\prime \prime \prime}(z)}{f^{\prime}(z)}-\frac{3}{2}\left(\frac{f^{\prime \prime}(z)}{f^{\prime}(z)}\right)^{2}=0
$$

for all complex $z$ satisfying $f^{\prime}(z) \neq 0$. Thus, the Schwarzian derivative of $f$ vanishes for all $z$ satisfying $f^{\prime}(z) \neq 0$. Therefore by a well known fact (cf. [4]), $f(z)$ is a Möbius transformation of $z$.

\section{ACKNOWLEDGMENTS}

Many thanks to the referee for the very helpful comments.

\section{REFERENCES}

1. H. S. M. Coxeter and S. L. Greitzer, Geometry Revisited, Random House, New York, 1967.

2. B. A. Fuchs and B. V. Shabat, Functions of a Complex Variable I, Pergamon Press, 1964. MR 28:4087

3. H. Haruki and Th. M. Rassias, A new characteristic of Möbius transformations by use of Apollonius quadrilaterals, Proc. Amer. Math. Soc. 126 (10) (1998), 2857-2861. CMP 99:04

4. H. Haruki, A proof of the principle of circle-transformation by the use of a theorem on univalent functions, L'Enseignement Mathematique 18 (2) (1972), 145-146. MR 48:4312]

5. S. Rabinowitz (ed.), Index to Mathematical Problems 1980-1984, MathPro Press, 1992.

Department of Pure Mathematics, Faculty of Mathematics, University of Waterloo, Waterloo, Ontario, Canada N2L 3G1

Department of Mathematics, National Technical University of Athens, Zografou Campus, 15780 Athens, Greece

E-mail address: trassias@math.ntua.gr 